\title{
Indiplon in the treatment of sleep disorders
}

\author{
Alan Lankford \\ Sleep Disorders Center of Georgia, \\ Atlanta, GA, USA
}

\begin{abstract}
Indiplon is a novel sedative-hypnotic recently approved for the treatment of insomnia. Like other non-benzodiazepine hypnotics, its mechanism of action is to modulate subunits, especially the alpha-1 subunit, of the GABA receptor complex in order to induce sedation. Indiplon was developed in two different formulations to address two different types of insomnia complaint: indiplon-IR (immediate release) was designed for sleep onset difficulties, while indiplon-MR (modified release) was developed for sleep maintenance insomnia. While there are currently few peer reviewed articles about indiplon clinical trial results, the early information that is available seems to indicate that both formulations have been well tolerated and have proven effective at improving both patient reported and objectively measured sleep parameters in both adult and elderly insomnia patients. In May 2006, the FDA indicated that indiplon-IR was approvable and plans are to resubmit the application in 2007. Indiplon-MR was unapprovable and may require further evaluation.
\end{abstract}

Keywords: indiplon, insomnia, $\mathrm{GABA}_{\mathrm{A}}$ receptor, sedative, non-benzodiazepine

\section{Introduction}

Insomnia is a common sleep disorder with potentially serious economic, health and quality of life ramifications if left untreated. It is characterized by symptoms of difficulty falling asleep, difficulty staying asleep, waking too early or poor quality or non-restorative sleep (American Psychiatric Association 2000; American Sleep Disorders Association 2001). Epidemiological studies have documented the prevalence of chronic insomnia in 10\%-15\% of the general population (Ford and Kamerow 1989; Ancoli-Israel and Roth 1999; National Sleep Foundation 2002, 2003, 2004, 2005; Drake et al 2003). Furthermore, as many as half of all Americans experience transient episodes of insomnia symptoms due to acute circumstances (National Sleep Foundation 2002, 2003, 2004, 2005).

Chronic insomnia is associated with significant daytime distress. Consequences of the disease can include functional impairment, increased risk of accidents and reductions in quality of life (Ford and Kamerow 1989; Balter and Uhlenhuth 1991; Rosenthal et al 1993; Zammit 1999). Specific medical conditions associated with insomnia include coronary artery disease, hypertension and musculoskeletal problems (Gislason et al 1993; Janson et al 2001; Mallon et al 2002). Additionally, numerous studies have established a remarkably high association between insomnia and psychiatric disorders, especially depression, other affective disorders and anxiety (Ford and Kamerow 1989; Benca et al 2004). Evidence of a bidirectional interaction between insomnia and other co-morbidities, specifically depression and some pain disorders is beginning to emerge (Moldofsky et al 1975; Moldofsky and Scarisbrick 1976). Across several studies lasting up to 35 years, insomnia has been shown to increase the risk for the development of depression by as much as a factor 5 (Ford 1989; Breslau 1996). Meanwhile, pain has been shown to exacerbate sleep difficulties, while poor sleep intensifies the perception of pain (Moldofsky et al 1975; Moldofsky and Scarisbrick 1976; Drewes et al 1997). 
While the underlying causes of insomnia are only partially understood, certain risk factors may predispose individuals to this disorder. These risk factors include age, gender, psychiatric or physical illness, the use of stimulants like caffeine and nicotine, some forms of shift work and poor sleep hygiene. Other potential contributors, including genetic etiology, are the focus of on-going research.

Recently, the serious consequences of insomnia have begun to receive more prominent attention. After a hiatus of 22 years, the National Institutes of Health convened a State of the Science conference on chronic insomnia in mid-2005 (National Institutes of Health 2005). Notably, the conference statement recommended that insomnia be considered as either a primary disorder or co-morbid with other disorders. Prior to this assertion, insomnia was typically considered to be a symptom secondary to other medical conditions and, as such, was often undertreated. Furthermore in the past few years, several new drugs were approved by the FDA for the treatment of insomnia. Greater national attention to the disorder and an expanded number of treatment options have resulted in marked changes in the pharmaceutical marketplace. Sales of prescription sleep aids in the U.S. have markedly increased from approximately $\$ 900 \mathrm{M}$ in 2000 to more than $\$ 2.1 \mathrm{~B}$ in 2004 (Iskowitz 2005).

Most of the currently available hypnotics are best suited to address difficulties with sleep onset (eg, difficulty falling asleep). Two of the newest treatment options, eszopiclone and zolpidem-CR, appear to assist patients with sleep maintenance difficulties (eg, nighttime awakenings). In order to minimize the potential for negative events associated with residual daytime sedation, all currently approved insomnia therapies are recommended to be taken prior to bedtime so that a patient can spend a full 8 hours in bed. As information about the health impact of insomnia is more widely disseminated and physicians continue to place more importance on its treatment, the number of patients taking prescription sleep medicine will continue to grow, as will the need for more specialized treatment options.

\section{Current treatment options}

Benzodiazepines were among the first modern drugs developed to treat the symptoms of insomnia. Benzodiazepines specifically approved for this purpose included flurazepam (Dalmane), temazepam (Restoril), triazolam (Halcion), quazepam (Doral) and estazolam (Prosom) (Eddy and Walbroehl 1999). This class of drug targets the GABA receptors and can produce sedative, hypnotic, anxiolytic, anticonvulsive, anesthetic and muscle-relaxant effects (Haefely et al 1992).
Unfortunately, most benzodiazepines interact with the entire GABA receptor complex, creating several of these effects simultaneously. Furthermore, short term use of benzodiazepines is recommended because of concerns about tolerance, dependence and the potential for significant adverse events (Kales et al 1983; Bixler et al 1987; Adams and Oswald 1989; Holbrook et al 2000).

More recently, treatment options have grown to include non-benzodiazepines and melatonin agonists. The off-label usage of other drug classes (eg, antidepressants, antihistamines) to treat insomnia complaints is also common, although there is limited evidence that these agents are safe and effective for use in this manner (Hartmann 1995; Nowell et al 1999; Richardson et al 2002).

In contrast to the benzodiazepines, the non-benzodiazepines are more selective for specific GABA receptor subunits associated with sedation. These compounds have a rapid onset of action and also rapidly eliminated from the body. Thus, they are able to quickly induce sedation with fewer of the next-day effects associated with some longer acting benzodiazepines (Nicholson et al 1983; Walsh et al 1990; Scharf et al 1994; Walsh et al 1998; Fry et al 2000). Non-benzodiazepines approved for the U.S. market include: zaleplon, zolpidem, zolpidem-CR and eszopiclone, the S-isomer of zopiclone. Zopiclone is widely used as a sedative outside the U.S.

At present, ramelteon is the only melatonin agonist approved for insomnia therapy. It acts on the $\mathrm{MT}_{1}$ and $\mathrm{MT}_{2}$ receptors which help maintain the circadian rhythms underlying the normal sleep-wake cycle (Brzezinski 1997; Hirai et al 2003). An important difference between ramelteon and both the benzodiazepines and non-benzodiazepines is that it has not been designated as a scheduled drug by the U.S. Drug Enforcement Agency due to its clinically established, extremely low potential for abuse (Hirai et al 2005; Griffiths et al 2005; France et al 2006). In addition, ramelteon is a completely different way to treat insomnia medically, focusing on the melatonin pathway rather than the GABA pathway.

Indiplon is one of the newest insomnia treatments in the non-benzodiazepine class. In contrast to other members of this class, two formulations of indiplon were simultaneously developed in order to address two distinct insomnia complaints. Indiplon-IR (immediate release) was designed to address sleep onset difficulties, both at the beginning of the night and after middle of the night awakenings. Indiplon-MR (modified release) was designed to address sleep maintenance difficulties. The FDA gave Neurocrine Biosciences an approvable letter for Indiplon-IR in May 2006 
and simultaneously stated that the approval of Indiplon-MR would require further discussions and potentially additional development efforts.

\section{Pharmacology of indiplon}

Indiplon is a novel pyrazolopyrimidine with the empiric formula $\mathrm{C}_{20} \mathrm{H}_{16} \mathrm{~N}_{4} \mathrm{O}_{2} \mathrm{~S}$. It is chemically described as $N$-Methyl- $N$-[3-[3-(thien-2-ylcarbonyl)pyrazolo[1,5 $\alpha]$ pyrimidin-7-yl]phenyl]acetamide.

Indiplon pharmacokinetics (PK) have been investigated in both animal and human studies. Healthy young male and female humans have demonstrated a maximal concentration $\left(\mathrm{C}_{\max }\right)$ of indiplon at 0.73 and 0.82 hours after dosing and exhibited an elimination half life $\left(\mathrm{t}_{1 / 2}\right)$ of 1.97 and 1.71 hours, respectively (Rogowski et al 2002). Even though slight gender differences were observed, they were not statistically significant. Another study comparing indiplon PK in healthy adult and elderly subjects found that indiplon's peak plasma concentration was reached in 2.3 hours and the elimination half life was 1.5 hours in the younger group, as compared to 2.7 hours and 1.8 hours in the older group (Jochelson et al 2002). Again, these differences did not prove to be statistically significant.

Differences in PK values due to gender and age are frequently observed with both other hypnotics. Maximum concentration, total exposure and half life can be up to $50 \%$ greater in women (Rosen et al 1999; Greenblatt et al 2000). In the elderly, impaired renal and hepatic function can lead to a 3-fold increase in plasma concentrations and a doubling of half-life relative to the values seen younger adults. PK differences due to gender have been associated with metabolization via the hepatic isoform CYP 3A4, while age differences have been linked to the cytochrome P450 metabolic pathway (Greenblatt et al 1991, 2000). Indiplon is metabolized equally by CYP $3 \mathrm{~A} 4$ and non-microsomal esterases, potentially explaining its relative lack of gender and age effects as compared to other hypnotics.

Of the currently available treatments, zaleplon is the only non-benzodiazepine hypnotic of the pyrazolopyrimidine class, the same chemical class as indiplon. Compared to indiplon, zaleplon has a somewhat shorter time to peak plasma concentration at approximately one hour as well as a shorter elimination half life of approximately one hour. The pharmacokinetics of zaleplon do not vary significantly with age or gender, however hepatic impairment can lead to a 4-7-fold increase in plasma concentration, a finding similar to indiplon.
While indiplon has not been shown to interact with alcohol, zaleplon potentiates the CNS-impairing effects for 1-2.5 hours after co-administration. Finally, zaleplon, like indiplon, shows no development of tolerance during short term nightly administration.

A significant difference between these two compounds is that indiplon is 10 times more selective for the GABA-A alpha 1 subunit, which is associated with sedation, compared to the alpha 2, 3 or 5 subunits. Zaleplon is only twice as selective for the alpha 1 subunit.

Co-administration of indiplon and alcohol does has not been shown to produce any pharmacokinetic or pharmacodynamic (PD) interactions (Berkowitz et al 2003). The absence of effect on PK values is expected since alcohol and indiplon are eliminated via different metabolic pathways. However, the absence of impact on PD parameters is noteworthy and unique relative to many other hypnotics.

Finally, the potential for indiplon to produce tolerance was evaluated in healthy volunteers (Jochelson, Chen et al 2003). Both PK and PD parameters were essentially unchanged between the first and last day of the two week study at all dose levels (10-40 mg), indicating no development of tolerance following repeat administration of indiplon.

\section{Indiplon in clinical trials}

Indiplon was developed by Neurocrine Biosciences in two distinct formulations. The immediate release form (-IR) was designed to address sleep onset difficulties, while the modified release form (-MR) was developed to help patients stay asleep throughout the entire night. The FDA gave Neurocrine an approvable letter for indiplon-IR 5 and $10 \mathrm{mg}$ in May 2006. Neurocrine plans to resubmit the application in mid-year 2007 after conducting further analyses and modifications to previous analyses. A supplemental pharmacokinetic/food effect profile was also requested by the FDA and has been completed. Indiplon-MR $15 \mathrm{mg}$ was found unapprovable. At the time of this review, Neurocrine may continue to evaluate the MR formulation for sleep maintenance insomnia.

Most of the currently available data on indiplon comes from either abstracts written by researchers involved in studies sponsored by Neurocrine or from corporate press releases. These studies are summarized in Table 1. Additional information from these trials will likely become available as the company begins the market launch of one or both formulations of the drug. 
Table I Indiplon (-IR and -MR) studies

\begin{tabular}{|c|c|c|c|}
\hline Authors & Patients (N) & Indiplon dosage & Duration \\
\hline Rogowski et al 2002 & Healthy adults (24) & IR: I5 mg & I day \\
\hline Berkowitz et al 2003 & Healthy adults $(10)$ & IR: 10 mg & I day \\
\hline Roth et al 2003 & Healthy adults (228) & IR: $15,30 \mathrm{mg}$ & I day \\
\hline Scharf et al 2004 & Healthy adults (593) & IR: I0, $20 \mathrm{mg}$ & I day \\
\hline Garber et al 2004 & Healthy adults (35) & IR: I0, 20 mg & I day \\
\hline Jochelson, Chen et al 2003 & Healthy adults (30) & IR: I0, 30, $45 \mathrm{mg}$ & 2 weeks \\
\hline Jochelson et al 2002 & Healthy adults and elderly (25) & IR: I5 mg & 4 days \\
\hline Neurocrine Biosciences 2003 & Healthy elderly (36) & IR: 5,10 mg & I day \\
\hline Roth et al 2005 & Chronic insomniacs (260) & IR: I0, 20 mg & 4 weeks \\
\hline Walsh et al 2004 & Chronic insomniacs (194) & IR: I0, $20 \mathrm{mg}$ & 5 weeks \\
\hline Scharf et al 2005 & Chronic insomniacs (702) & IR: I0, $20 \mathrm{mg}$ & 3 months \\
\hline Black et al 2006 & Chronic insomniacs (536) & IR: I0, 20 mg & 12 months \\
\hline Scharf et al 2003 & Elderly chronic insomniacs (42) & IR: 5, I0, $20 \mathrm{mg}$ & I day \\
\hline Walsh et al 2005 & Elderly chronic insomniacs (358) & IR: 5,10 mg & 2 weeks \\
\hline Moscovitch et al 2006 & Elderly chronic insomniacs ( $12 \mid$ ) & IR: $5,10 \mathrm{mg}$ & 6 months \\
\hline Corrigan et al 2006 & Healthy adults & IR: up to $30 \mathrm{mg}$ & various \\
\hline Cohn et al 2004 & Healthy adults (I2) & MR: 30 mg & I day \\
\hline Jochelson, Bozigian et al 2003 & Healthy adults (36) & MR: 40 mg & I day \\
\hline Neurocrine Biosciences 2004d & Healthy adults (325) & MR: 20, $30 \mathrm{mg}$ & I day \\
\hline Hull et al 2004 & $\begin{array}{l}\text { Adults with mild to moderate } \\
\text { chronic obstructive } \\
\text { pulmonary disease (18) }\end{array}$ & MR: 20 mg & I day \\
\hline Jochelson et al 2004 & Chronic insomniacs (2II) & MR: $30 \mathrm{mg}$ & 2 weeks \\
\hline Klee et al 2006 & Chronic insomniacs (248) & MR: I5 mg & 4 weeks \\
\hline Neurocrine Biosciences 2004b & Chronic insomniacs (740) & MR: 20, 30 mg & 3 months \\
\hline Rosenberg et al 2006 & $\begin{array}{l}\text { Adult and elderly chronic } \\
\text { insomniacs }(100)\end{array}$ & MR: I5 mg & 2 days \\
\hline Walsh et al 2003 & Elderly chronic insomniacs (60) & MR: I0, 20,30, $35 \mathrm{mg}$ & 2 days \\
\hline Lankford et al 2005 & Elderly chronic insomniacs (229) & MR: I5 mg & 2 weeks \\
\hline Neurocrine Biosciences 2004c & Elderly chronic insomniacs (220) & MR: $20 \mathrm{mg}$ & 2 weeks \\
\hline Neurocrine Biosciences 2004a & Elderly chronic insomniacs (340) & MR: 20, $30 \mathrm{mg}$ & 5 weeks \\
\hline
\end{tabular}

\section{Indiplon-IR}

Indiplon-IR has been studied in both healthy volunteers and in patients meeting the Diagnostic and Statistical Manual of Mental Disorders $\left(\mathrm{DSM}-\mathrm{IV}^{\circledR}{ }^{\circledR}\right.$ criteria for primary insomnia for at least three months (American Psychiatric Association 2000). Two studies induced transient insomnia in healthy adults via a 2-hour phase advance and the sleep laboratory "first night effect." The impact of indiplon-IR relative to placebo was objectively measures using polysomnography (PSG) in both of these trials (Roth et al 2003; Scharf et al 2004). Objective Latency to Persistent Sleep (LPS) and patient reported Latency to Sleep Onset (LSO) were both significantly improved with all four doses evaluated (10 and $20 \mathrm{mg}$ in the 1 st study, 15 and $30 \mathrm{mg}$ in the 2nd). The first study also found that indiplon-IR produced significant improvements in objective and subjective Total Sleep Time (TST, sTST), sleep efficiency and several measures of sleep quality.

Two placebo-controlled trials studied the effects of indiplon-IR (10 and $20 \mathrm{mg}$ ) in adults with primary insomnia. The first trial used PSG to measure patient sleep parameters at the beginning of the first, third and fifth weeks of treatment (Walsh et al 2004). Relative to placebo, both doses of indiplon significantly reduced LPS and patient reports of LSO were also significantly improved at weeks 1 and 5 . Significant improvements were also seen in TST $(10 \mathrm{mg}$ only) and sleep quality (both doses) at week 1 . The second study evaluated subjective sleep parameters over the course of three months (Scharf et al 2005). Patients in this trial reported that indiplon-IR significantly improved the time to sleep onset, sTST, subjective Wake time After Sleep Onset (sWASO) and sleep quality at all time points. A subset of the study population continued treatment for up to 6 months. Improvements in sleep onset were maintained throughout this period of time.

Indiplon-IR was evaluated in two trials with elderly insomnia patients. The first was a dose response study comparing the effects of 5,10 and $20 \mathrm{mg}$ of indiplon-IR with placebo (Scharf et al 2003). All doses were shown to produce significant improvements in LPS, LSO, sTST and sleep quality. The second study used patient reports to evaluate 
the effects of 5 and $10 \mathrm{mg}$ of indiplon relative to placebo (Walsh et al 2005). Both dose levels produced significant improvements in LSO, sTST, the subjective Number of Awakenings (sNAW) and sleep quality during throughout the 2 week treatment period. Additionally, the $10 \mathrm{mg}$ dose group showed significantly improvement in sWASO at both time points. Again, treatment was continued for up to 6 months for a subset of the patients in this trial (Moscovitch et al 2006). Over this extended timeframe, $81 \%$ of the study participants randomized to indiplon-IR reported that it improved their sleep ( $72 \%$ on $5 \mathrm{mg}, 92 \%$ on $10 \mathrm{mg}$ ).

\section{Indiplon-MR}

Indiplon-MR is an extended release formulation that delivers part of its dose initially and then provides a controlled release of the remainder over the course of the night. It has been tested in studies involving healthy volunteers and in insomnia patients. Transient insomnia was induced in healthy adults via the sleep laboratory model of the "first night effect" and a 2-hour phase advance (Neurocrine Biosciences 2004d). As compared to placebo, PSG measured LPS and subjective LSO were significantly reduced for both doses studied (20 and $30 \mathrm{mg}$ ). A second study in healthy adults used repeated venipunctures to simulate sleep maintenance difficulties (Jochelson, Bozigian et al 2003). In this evaluation, indiplon-MR $40 \mathrm{mg}$ significantly improved LSO, sTST and sleep quality relative to placebo. The subjective number of awakenings declined non-significantly, likely due to the stimulus created by multiple instances of blood sampling.

All other indiplon-MR studies were performed with insomnia sufferers, including three trials using adult patients. The first of these three studies randomized patients to either $30 \mathrm{mg}$ of indiplon-MR or placebo (Jochelson et al 2004). Sleep continuity, as measured by sTST, sWASO and sNAW, was significantly improved during the two weeks of treatment with indiplon. Sleep onset and quality also showed significantly improvements at all time points.

The second study lasted for three months and treated the adult patients with either indiplon-MR (20 or $30 \mathrm{mg}$ ) or placebo (Neurocrine Biosciences 2004b). Again, measures of sleep maintenance (sTST, subjective Total Wake Time (sTWT), sWASO and sNAW) were significantly improved for both doses over the length of the trial. Participants also reported significant improvements in sleep quality and LSO. The third and final trial in adults compared the indiplon-MR dose submitted for FDA approval $(15 \mathrm{mg}$ ) with placebo (Klee et al 2006). Patient reports demonstrated significant improvements in sTST, sWASO, sNAW, sTWT, LSO and sleep quality over the course of the 4 week long study.

Both adult and elderly insomnia patients participated in a placebo-controlled, cross-over PSG study of indiplon-MR $15 \mathrm{mg}$ (Rosenberg et al 2006). Active treatment produced significant improvements in all objective measures of sleep maintenance (WTDS, WASO and TST) and sleep onset. LSO and sleep quality were also significantly improved. Notably, at present this is the only study in the public domain that uses PSG to objectively evaluate indiplon-MR $15 \mathrm{mg}$ in adult patients - the dose submitted to the FDA for approval.

Indiplon-MR has been tested in four trials with elderly insomnia patients. The first of these was a PSG dose-response study comparing indiplon-MR 10, 20, 30 and $35 \mathrm{mg}$ with placebo (Walsh et al 2003). In this trial, significant improvements in WASO and mean sleep efficiency were seen for indiplon-MR doses of $20 \mathrm{mg}$ and above. Relative to placebo, significant improvements in LPS and NAW were demonstrated by all dose levels of active treatment.

Elderly chronic insomnia patients with sleep maintenance difficulties participated in a two week trial of indiplon-MR $20 \mathrm{mg}$ (Neurocrine Biosciences 2004c). At all time points, both sTST and sWASO were significantly improved with treatment. Two investigator global rating scales, subjective sleep maintenance endpoints (sTWT, sNAW), sleep onset and quality were also significantly improved relative to placebo. In another study with elderly patients, indiplon-MR 20 and $30 \mathrm{mg}$ were compared with placebo over a period of 5 weeks (Neurocrine Biosciences 2004a). PSG measured sleep maintenance (WASO, TST, WTDS, and sleep efficiency) and onset (LPS) were significantly improved at all time points. Furthermore, relative to those on placebo, indiplon-MR patients also reported that their sleep quality improved significantly.

Finally, the indiplon-MR dose submitted to the FDA for approval (15 mg) was evaluated in 229 elderly insomnia patients (Lankford et al 2005; Lydiard et al 2006) in a manner similar to that of the adult insomnia patient trial of indiplon-MR $15 \mathrm{mg}$ previously mentioned (Klee et al 2006). As in the adult patient study, the participants in this evaluation reported significant improvement in all sleep measures at all time points. Measures evaluated included sTST, LSO, sWASO, sNAW, sTWT and sleep quality.

\section{Safety and tolerability of indiplon}

Indiplon's safety and tolerability has been assessed in all of the studies reviewed. Overall, few adverse events were 
produced by either formulation, and those that did occur were principally consistent with the known effects of hypnotics.

Adverse events were evaluated over the course of a 12 month trial in adult insomnia patients randomized to placebo or indiplon-IR 10 or $20 \mathrm{mg}$ (Black et al 2006). The most frequent adverse events seen at the $20 \mathrm{mg}$ dose level were headaches $(8.7 \%)$, URI $(6.5 \%$ and nasopharyngitis (5.9\%). The $10 \mathrm{mg}$ group reported headaches (11.8\%), back pain and somnolence (both 7.9\%). Over the course of the study no clinically relevant changes in vital signs or ECG parameters occurred.

Several pharmacodynamic tests were used across multiple trials to measure the next-day effects of indiplon. The Visual Analog Scales of sleepiness (VAS) was used to measure next day sedation, while the Digit Symbol Substitution Test (DSST) and Symbol Copying Test (SCT) were both used to evaluate residual functional impairment. Across four trials (Roth et al 2003; Scharf et al 2003, 2004; Walsh et al 2004) no changes in any of these next-day measures was observed at any dose level of indiplonIR studied (5 to $30 \mathrm{mg}$ ). Evaluations of indiplon-MR's residual effects have been reported for two trials. Only the highest dose evaluated $(35 \mathrm{mg}$ ) was shown to produce a modest effect on DSST scores (Walsh et al 2003; Lydiard et al 2006). No other impairment or evidence of residual sedation was found.

The potential for the development of tolerance and withdrawal symptoms resulting from indiplon was examined across several studies. A 2-week repeat dosing study in healthy volunteers found no evidence of PK or PD tolerance with indiplon-IR (Jochelson, Chen et al 2003). Three additional trials in insomnia sufferers incorporated the Benzodiazepine Withdrawal Symptom Questionnaire (Walsh et al 2004, 2005; Scharf et al 2005). Using this scale, no signs of withdrawal were found during treatment that lasted up to 3 months with indiplon-IR 5 to $20 \mathrm{mg}$.

In spite of warnings against doing so, insomnia patients often consume hypnotics and alcohol together. Coadministration of these compounds often leads to changes in drug PK which may, in turn, result in additive impairment. The interaction of indiplon-IR and alcohol was evaluated to assess the likelihood of these effects (Berkowitz et al 2003). This study found a small, non-additive reduction in performance on pharmacodynamic tests (DSST and SCT). Reaction time and sedation (VAS) were unaffected.

Studies of healthy volunteers have assessed the potential for drug-drug interactions between indiplon and several other compounds (Corrigan et al 2006). These evaluations have found that indiplon does not impact the PK of either digoxin or theophylline. The PK and PD of warfarin also remained unaffected. Co-administration of indiplon with antidepressants (sertraline, paroxetine, venlafaxine, amitriptyline) does not result in additive effects on PD assessments and appears to have no effect on indiplon's PK values.

Respiratory depression in individuals with chronic obstructive pulmonary disease (COPD) and sleep apnea has been observed when individuals from these at-risk group use benzodiazepine hypnotics. Unfortunately, sleep apnea and COPD are strongly linked with co-morbid insomnia, creating complex treatment trade-offs (Fleetham et al 1982; Weiss et al 2000). Indiplon-MR's respiratory depressant effects were assessed in COPD patients to evaluate the risk indiplon presents for this patient subset (Hull et al 2004). Relative to placebo, no changes in either the arterial oxygen saturation level or the respiratory disturbance index were produced by single night of indiplon-MR $20 \mathrm{mg}$. Additionally, half of the individuals receiving indiplon reported "very good" or "excellent" sleep quality vs only $17 \%$ of those randomized to placebo. Indiplon-MR $30 \mathrm{mg}$ was compared to codeine sulfate $60 \mathrm{mg}$ (active control) and placebo in a daytime $\mathrm{CO}_{2}$ challenge model (Cohn et al 2004). After dosing with codeine, healthy volunteers experienced respiratory suppression for 8 hours. In contrast, no respiratory effects were demonstrated at any time point following administration of indiplon.

Finally, middle of the night dosing with indiplon-IR was evaluated. Existing therapeutics are not appropriate for insomniacs who awaken in the middle of the night and have difficulties returning to sleep because these medications' relatively long duration of action can produce significant next day impairments when taken with less than 8 hours available time in bed. The next-day effects of taking indiplon-IR with only 4 hours remaining time in bed were studied in three separate trials. Two of these trials also incorporated other hypnotics as comparators.

A single-night, cross-over study in healthy adults woke participants after they had spent only 4 hours in bed (Garber et al 2004). Volunteers were dosed with either indiplon-IR (10 or $20 \mathrm{mg}$ ), zolpidem $10 \mathrm{mg}$, zopiclone $7.5 \mathrm{mg}$ or placebo and were then permitted to remain in bed for another 4 hours before being awakened a second time. Participants performed the DSST, SCT and VAS at 4 and 6 hours postdose to assess the relative next-day residual effects of study treatment. Relative to placebo, indiplon-IR was the only drug that did not produce any significant changes on any of these tests. Zolpidem produced a significant increase in 
sleepiness at 4 hours post-dose and significantly increased sedation was seen at both 4 and 6 hours post-dose with zopiclone.

Healthy elderly volunteers participated in a similar trial comparing the effects of 5 and $10 \mathrm{mg}$ of indiplon-IR, following middle of the night dosing, with those resulting from $3.75 \mathrm{mg}$ of zopiclone and placebo (Neurocrine Biosciences 2003). As in the study with young adults, neither dose of indiplon-IR produced any significant changes on the DSST, SCT or VAS at any time point. Participants randomized to zopiclone, however, demonstrated significant changes on the DSST at 4 and 8 hours post-dose and showed a trend toward impairment on the SCT at both 4 and 6 hours post-dose.

The third study of middle of the night dosing was conducted in adult insomnia patients on an outpatient basis (Roth et al 2005). Over the course of 4 weeks, double-blind therapy (indiplon-IR 10 or $20 \mathrm{mg}$, placebo) was self administered on a prn basis as long as at least 4 hours sleep time was available to the patient. Participants reported that LSO post-dose was significantly shortened at all time points. Furthermore, over the course of the study, the average sTST was significantly extended for patients randomized to both doses of indiplon-IR relative to those treated with placebo. VAS ratings showed no increase in next day sleepiness at either dose of active treatment.

\section{Summary}

Indiplon is a novel sedative-hypnotic that modulates subunits of the GABA receptor complex in order to induce sedation. It has been shown to be well tolerated and effective as an insomnia therapy in randomized, placebo-controlled trials involving more than 7,500 participants. Both patient reported and objectively measured sleep parameters have shown consistent improvements without next-day residual sedation or impairment. Indiplon was developed in two different formulations to address two different types of insomnia complaint: indiplon-IR (immediate release) was designed for sleep onset difficulties, while indiplon-MR (modified release) was developed for sleep maintenance insomnia. IndiplonIR shows potential for use on a prn basis, for example, by patients who wake in the middle of the night and are unable to return to sleep without assistance.

\section{Disclosures}

Dr Lankford has been an investigator in clinical trials covered in this review. He has also served on an advisory board for Neurocrine Biosciences.

\section{References}

Adams K, Oswald I. 1989. Can a rapidly-eliminated hypnotic cause daytime anxiety? Pharmacopsychiatry, 22:115-19.

Ancoli-Israel S, Roth T. 1999. Characteristics of insomnia in the United States: results of the 1991 National Sleep Foundation Survey. Sleep, 22:S347-53.

American Psychiatric Association. 2000. Diagnostic and Statistical Manual of Mental Disorders - 4th edition, text revision. Washington D.C.

American Sleep Disorders Association. 2001. The international classification of sleep disorders: Diagnostic and coding manual. Rochester MN.

Balter MB, Uhlenhuth EH. 1991. The beneficial and adverse effects of hypnotics. J Clin Psychiatry, 52(7 Suppl):16-23.

Benca RM, Ancoli-Israel A, Moldofsky H. 2004. Special considerations in insomnia diagnosis and management: depressed, elderly and chronic pain populations. J Clin Psychiarty, 65(Suppl 8):26-35.

Berkowitz DV, Scharf MB, Jochelson P, et al. 2003. The co-administration of indiplon (NBI-34060) and alcohol lacks pharmacokinetic and pharmacodynamic interaction. Sleep, 26:A81 (Abstract supplement).

Bixler EO, Kales A, Burbaker BH, et al. 1987. Adverse reactions to benzodiazepine hypnotics: spontaneous reporting system. Pharmacology, 35:286-300

Black JE, Burke J, Bell J, et al. 2006. Safety and tolerability of long-term treatment with indiplon: results of a randomized 12-month study. Presented at American Psychological Association conference, Toronto.

Breslau N, Roth T, Rosenthal L, et al. 1996. Sleep disturbance and psychiatric disorders: a longitudinal epidemiological study of young adults. Biol Psychiatry, 39:411-18.

Brzezinski A. 1997. Melatonin in humans. N Engl J Med, 336:186-95.

Cohn M, Jochelson P, Gately N, et al. 2004. Assessment of respiratory effects of indiplon-MR in a $\mathrm{CO}_{2}$ challenge model. Sleep, 27:A261 (Abstract supplement).

Corrigan B, Hershberger E, Abdulnabi R, et al. 2006. Evaluation of drugdrug interactions with indiplon. Presented at American Psychological Association conference, Toronto.

Drake CL, Roehrs T, Roth T. 2003. Insomnia causes, consequences, and therapeutics: an overview. Depress Anxiety, 18:163-76.

Drewes AM, Nielson KD, Arendt-Nielsen L, et al. 1997. The effect of cutaneous and deep pain on the electroencephalogram during sleep: an experimental study. Sleep, 20:632-40.

Eddy M, Walbroehl GS. 1999. Insomnia. Am Fam Physician, 59:1911-18.

Fleetham J, West P, Mazon B, et al. 1982. Sleep, arousals and oxygen desaturation in chronic obstructive pulmonary disease. Am Rev Respir Dis, 126:429-33.

Ford DE, Kamerow DB. 1989. Epidemiologic study of sleep disturbances and psychiatric disorders: an opportunity for prevention? JAMA, 262:1479-84.

France CP, Weltman RH, Koek W, et al. 2006. Acute and chronic effects of ramelteon in rhesus monkeys (Macaca mulatta): dependence liability studies. Behav Neurosci, 120:535-41.

Fry J, Scharf M, Mangano R, et al. 2000. Zaleplon improves sleep without producing rebound effects in outpatients with insomnia. Int Clin Psychopharmacol, 15:141-52.

Garber M, Burke J, Farber R, et al. 2004. Residual effects of middle of the night dosing: a placebo-controlled crossover study of indiplon-IR, zolpidem, and zolpiclone in healthy volunteers. Sleep, 27:A260 (Abstract supplement).

Gislason T, Reynisdottir H, Kristbjarnarson H, et al. 1993. Sleep habits and sleep disturbances among the elderly - an epidemiological survey. J Int Med, 234:31-9.

Greenblatt DJ, Harmatz JS, Von Moltke LL, et al. 2000. Comparative kinetics and response to the benzodiazepine agonist triazolam and zolpidem; evaluation of sex-dependent differences. J Pharmacol Exper Ther, 293:435-43.

Greenblatt DJ, Harmatz JS, Shader RI. 1991. Clinical pharmacokinetics of antiolytics and hypnotics in the elderly. Clin Pharmacokinet, 21:262-73. 
Griffiths R, Suess P, Johnson M. 2005. Ramelteon and triazolam in humans: behavioral effects and abuse potential. Sleep, 28(Suppl):A44. Abstract 0132 .

Haefely W, Facklam M, Schoch P, et al. 1992. Partial agonists of benzodiazepine receptors for the treatment of epilepsy, sleep and anxiety disorders. Adv Biochem Psychopharmacol, 47:379-94.

Hartmann PM. 1995. Drug treatment on insomnia: indications and newer agents. Am Fam Physician, 51:191-8.

Hirai K, Kita M, Ohta H, et al. 2005. Ramelteon (TAK-375) accelerates reentrainment of circadian rhythm after a phase advance of the lightdark cycle in rats. J Biol Rhythms, 20:27-37.

Hirai K, Kato K, Nishiyama K, et al. 2003. TAK-375 and its metabolites are selective agonists at ML-1 receptors. Sleep, 26(Suppl):A79.

Holbrook AM, Crowther R, Lotter A, et al. 2000. Meta-analysis of benzodiazepine use in the treatment of insomnia. CMAJ, 162:225-33.

Hull S, Fogarty C, Vince B, et al. 2004. Safety of indiplon-MR in patients with mild-to-moderate COPD. Sleep, 27:A261-2 (Abstract supplement).

Iskowitz M. 2005. Sleeping giants. MM and $M$, Nov:58-9.

Janson C, Lindberg E, Gislason T, et al. 2001. Insomnia in men - a 10-year prospective population based study. Sleep, 24:425-30.

Jochelson P, Scharf M, Roth T, et al. 2004. Efficacy of indiplon in inducing and maintaining sleep in patients with chronic sleep maintenance insomnia. Sleep, 27:A262 (Abstract supplement).

Jochelson P, Chen TK, Farber R, et al. 2003. Lack of pharmacological and pharmacokinetic tolerance following repeat dosing of Indiplon (NBI-34060). Sleep, 26:A85-6 (Abstract supplement).

Jochelson P, Bozigian H, Garber M, et al. 2003. The activity of modified release indiplon (NBI-34060) in a transient nighttime venipuncture model. Sleep, 26:A84 (Abstract supplement).

Jochelson P, Rogowski R, Burke J, et al. 2002. The pharmacokinetics of the non-benzodiazepine sedative-hypnotic, NBI-34060 in elderly subjects. Sleep, 25:A415 (Abstract supplement).

Kales A, Soldatos CR, Bixler EO, et al. 1983. Rebound insomnia and rebound anxiety: a review. Pharmacology, 26:121-37.

Klee B, Lankford DA, Landin R, et al. 2006. Sleep maintenance effect of indiplon in primary insomnia: results from a double-blind, placebocontrolled 4-week trial. Presented at American Academy of Neurology conference, New York.

Lankford J, Lydiard R, Walsh JK, et al. 2005. Efficacy and tolerability of indiplon-MR in elderly patients with chronic Insomnia: results of a double-blind, placebo-controlled, 2-week trial. Sleep, 28:A230 (Abstract supplement).

Lydiard RB, Lankford DA, Seiden DJ, et al. 2006. Efficacy and tolerability of modified-release indiplon in elderly patients with chronic insomnia: results of a 2-week double-blind, placebo-controlled trial. J Clin Sleep $\mathrm{Med}$, in press.

Mallon L, Broman JE, Hetta J. 2002. Sleep complaints predicts coronary artery disease mortality in males: a 12-year follow-up study of a middleaged Swedish population. J Intern Med, 251:207-16.

Moldofsky H, Scarisbrick P. 1976. Induction of neurasthenic musculoskeletal pain syndrome by selective sleep stage deprivation. Psychosom Med, 38:35-44.

Moldofsky H, Scarisbrick P, England R, et al. 1975. Musculosketal symptoms and non-REM sleep disturbance in patients with "fibrositis syndrome" and healthy subjects. Psychosom Med, 37:341-51.

Moscovitch A, Burke J, Farber R. 2006. Six month evaluation of indiplon in elderly chronic insomnia patients. Presented at American Association of Geriatric Psychiatry conference, San Juan Puerto Rico.

National Institutes of Health. State of the Science Conference Statement on Manifestations and Management of Chronic Insomnia in Adults [online]. Accessed 5/10/06. URL: http://consensus.nih.gov/2005/ 2005InsomniaSOS026html.htm

National Sleep Foundation. Sleep in America poll [online]. Data from 2002, 2003, 2004 and 2005 sleep polls. Accessed 5/10/06. URL: http://www. sleepfoundation.org/hottopics/index.php?secid=16
Neurocrine Biosciences: Press release - 2004a. 4/7/04: Neurocrine reports two additional phase III trials demonstrating indiplon is safe and effective in treating elderly patients with difficulties initiating and maintaining sleep. Accessed 5/22/06. http://phx.corporate-ir.net/phoenix. zhtml? $=68817 \& \mathrm{p}=1$ rol-newsArticle \&ID $=613211 \&$ highllght $=$

Neurocrine Biosciences: Press release - 2004b. 3/24/04: Neurocrine's 'SLEEP' study demonstrates highly positive long-term efficacy results with indiplon MR. Accessed 5/20/06. http://phx.corporate-ir.net/phoenix.zhtml?c=68817\&p=1rol-newsArticle \&ID= 613212\&highllght=

Neurocrine Biosciences: Press release - 2004c. 3/2/04: Neurocrine reports indiplon effective in treating elderly patients with difficulties maintaining sleep. Accessed 5/20/06. http://phx.corporate-ir.net/phoenix. zhtml?c=68817\&p=lrol-newsArticle \&ID=613218\&highllght=

Neurocrine Biosciences: Press release - 2004d. 3/1/04: Neurocrine reports positive results for indiplon in adults with transient insomnia. Accessed 5/18/06. http://phx.corporate-ir.net/phoenix.zhtml?c=68817\&p=1rolnewsArticle \&ID $=613219$ \& highllght $=$

Neurocrine Biosciences: Press release - 11/10/03, Neurocrine reports phase I clinical results with indiplon in elderly subjects after middle of the night dosing. Accessed 5/22/06. http://phx.corporate-ir.net/phoenix. zhtml? $=68817 \& \mathrm{p}=1$ rol-newsArticle\&ID=613229\&highllght $=$

Nicholson AN, Schlosberg A, Dreyfus JF. 1983. Zopiclone, a thirdgeneration of hypnotics. Pharmacology, 27(Suppl 2):165-215.

Nowell PD, Reynolds CF 3rd, Buysse DJ, et al. 1999. Paroxetine in the treatment of primary insomnia: preliminary clinical and electroencephalogram sleep data. J Clin Psychiatry, 60:89-95.

Physicians Desk Reference. 2007. Montvale, New Jersey: Thomson PDR.

Richardson GS, Roehrs RA, Rosenthal L, et al. 2002. Tolerance to daytime sedative effects of $\mathrm{H} 1$ antihistamines. J Clin Psychopharmacol, 22:511-15.

Rogowski R, Garber M, Bozigian H, et al. 2002. NBI-34060 (a nonbenzodiazepine sedative-hypnotic): lack of a pharmacokinetic gender effect. Sleep, 25:A415 (Abstract supplement).

Rosen AS, Fournie P, Darwish M, et al. 1999. Zaleplon pharmacokinetics and absolute bioavailability. Biopharm Drug Dispos, 20:171-5.

Rosenberg R, Hull S, Cohn M, et al. 2006. Sleep laboratory assessment of indiplon in primary insomnia: results of a double-blind, placebocontrolled, crossover trial. Presented at American Psychological Association conference, Toronto.

Rosenthal L, Roehrs TA, Rosen A, et al. 1993. Level of sleepiness and total sleep time following various time in bed conditions. Sleep, 16:226-32.

Roth T, Ancoli-Israel S. 1999. Daytime consequences and correlates of insomnia in the United States: results of the 1991 National Sleep Foundation survey. Sleep, 22(Suppl 2):S354-8.

Roth T, Walsh JK, Rogowski R, et al. 2003. Efficacy and tolerability of indiplon (NBI-34060) solution in healthy adults In a model on transient insomnia. Sleep, 26:A87 (Abstract supplement).

Roth T, Zammit G, Scharf MB, et al. 2005. Efficacy and safety of indiplon-IR in adults with chronic insomnia characterized by prolonged nighttime awakenings with difficulty returning to sleep. Sleep, 28:A229 (Abstract supplement).

Scharf MB, Black J, Hull S, et al. 2005. Long term efficacy and tolerability of indiplon-IR in the treatment of chronic insomnia: results of a double-blind, placebo-controlled 3-month study. Sleep, 28:A229 (Abstract supplement).

Scharf MF, Rosenberg R, Cohn M, et al. 2003. Safety and efficacy of immediate release indiplon (NBI-34060) in elderly patients with insomnia. Sleep, 26:A85 (Abstract supplement).

Scharf MB, Roth T, Rosenberg R, et al. 2004. Efficacy and tolerability of indiplon IR in the treatment of transient Insomnia. Sleep, 27:A267 (Abstract supplement).

Scharf MB, Roth T, Vogel G, et al. 1994. A multicenter placebo controlled study evaluating zolpidem in the treatment of chronic insomnia. J Clin Psychiatry, 55(5):192-9. 
Walsh JK, Fry J, Erwin CW, et al. 1998. Efficacy and tolerability of 14-day administration of zaleplon $5 \mathrm{mg}$ and $10 \mathrm{mg}$ for the treatment of primary insomnia. Clin Drug Invest, 16:347-54.

Walsh JK, Lankford DD, Krystal A, et al. 2003. Efficacy and tolerability of four doses of indiplon (NBI-34060) modified-release in elderly patients with sleep maintenance Insomnia. Sleep, 26:A78 (Abstract supplement).

Walsh JK, Rosenberg R, Roth T, et al. 2004. Treatment of primary insomnia for five weeks with indiplon-IR. Sleep, 27:A259 (Abstract supplement).

Walsh JK, Roth T, Moscovitch A, et al. 2005. Efficacy and tolerability of indlplon-IR in elderly patients with primary insomnia. Sleep, 28:A229 (Abstract supplement).
Walsh JK, Schweitzer PK, Sugerman JL, et al. 1990. Transient insomnia associated with a three-hour phase advance of sleeptime and treatment with zolpidem. J Clin Psychopharmacol, 10:184-9.

Weiss JW, Launois SH, Anand A. 2000. Cardiorespiratory changes in sleep-disordered breathing. In: Kryger MH, Roth T, Dement WC. eds. Principles and Practice of Sleep Medicine. 3rd ed. Philadelphia, PA: W.B. Saunders Co. p 859-68.

Zammit G. 1999. Insomnia interventions: new ideas for the management of sleep disorders. Clin Geriatr, July (Suppl):11-13. 
